\title{
KONGRESSKALENDER
}

\section{März 2019}

06.-08.03.2019, Lippstadt-Eickelborn 34. Eickelborner Fachtagung zu Fragen der Forensischen Psychiatrie Therapie und Sicherheit in der forensischen Psychiatrie Kontaktadresse: Michaela.Riepe@ wkp-lwl.org

Information: www.lwl-forensik-lippstadt.de

20.-22.03.2019, Potsdam-Griebnitzsee

20. Basiskurs Forensische Psychiatrie: Schwerpunkt Kriminalprognose anhand der neuen „Empfehlungen“

Prof. Dr. H.-L. Kröber, Dr. F. Wendt

Zentrum für Forensisch-Psychiatrische

Begutachtung

Information: www.zfpb.de; kroeber@zfpb.de

29.03.2019, Tübingen

Forensisch-Psychiatrisches Gespräch 2019: Umgang mit devianter Sexualität Psychiatrische Universitätsklinik, Hörsaal UKPP

Dr. Stephan Bork

Information: christel.weimer@

med.uni-tuebingen.de

\section{Mai 2019}

31.05.2019, Dresden

24. Dresdner Frühjahrstagung für

Forensische Psychiatrie

Körperlich begründbare Psychosen

- Klinik, forensische Bedeutung und sachverständige Beurteilung

Dr. Lange, Prof. Dr. Bauer, Dr. Lammel, Dr. Sutarski

Information: www.forensik-dresden.de

\section{Juni 2019}

05.-07.06.2019, Zürich, Schweiz

11. Internationales Symposium Forensische Psychiatrie

World Trade Center

Prof. Endrass, Dr. Rossegger, Prof. Urbaniok

Information: www.forensiktagung.ch

10.-12.06.2019, Stockholm, Schweden

The Stockholm Criminology Symposium

Swedish National Council of Crime

Prevention

City Conference Center, Stockholm

Information: www.criminologysymposium.

com
21.06.2019, Berlin-Mitte

23. Berliner Junitagung für Forensische Psychiatrie und Psychologie

Gibt es ,Kriminaltherapie“"? Die

Behandlung von Straffälligen auf dem Prüfstand

Forensische Psychiatrie Psychologie Berlin e. V. (FPPB) \& Institut für Forensische Psychiatrie der Charité

Langenbeck-Virchow-Haus

Information: www.forensik-berlin.de

(Veranstaltungen)

25.-27.06.2019, Montréal, Kanada

19th Annual IAFMHS Conference

Forensic Mental Health Services

Hotel Bonaventure

Information: www.iafmhs.org

\section{Juli 2019}

17.-20.07.2019, Santiago de Compostela, Spanien

European Association of Psychology and Law (EAPL)

29th Annual Conference

Prof. Ramón Arce

Information: www.sepjf.prg/eap12019

\section{September 2019}

\section{1.-13.09.2019, Hamburg}

Arbeitskreis Sozialtherapeutische

Anstalten im Justizvollzug e.V.

Überregionale Fachtagung

Information: http://sotha.de/de/tagungen/ fachtagung_sotha/2019_hamburg.html

18.-21.09.2019, Gent, Belgien

European Association of Criminology

19th Annual ESC Conference

Information: http://www.esc-eurocrim.org/

26.-28.09.2019, Hildesheim

Fachgruppe Rechtspsychologie der DGPs

\section{Fachtagung}

Die Vollzugsanstalt als Entwicklungsraum

Universität Hildesheim

Information: https://www.uni-hildesheim.de/ rechtspsychologie2019

28.-30.09.2019, Wien

16. Wissenschaftliche Fachtagung der

Kriminologischen Gesellschaft (KrimG)

Prof. Dr. Chr. Grafl

Information: www.krimg.de

\section{Oktober 2019}

Oktober 2019, München

34. Herbsttagung für Forensische

Psychiatrie

Abteilung für Forensische Psychiatrie der

Universität München

Prof. Dr. Schiltz

Information: www.forensik-muenchen.de

24.-27.10.2019, Baltimore, Maryland

(USA)

American Academy of Psychiatry and the Law

50th Annual Meeting (with APA)

Marriott Waterfront

Information: www.AAPL.org

\section{November 2019}

06.-09.11.2019, Atlanta, Georgia (USA) Association for the Treatment of Sexual Abusers (ATSA)

38th Annual Research and Treatment Conference

Hyatt Regency Atlanta

Information: http://www.atsa.com/futureatsa-conferences

13.-16.11.2019 San Francisco, California (USA)

American Society of Criminology -

Annual Meeting

San Francisco Marriott Marquis

Information: www.asc41.com

Hinweise für den Kongresskalender bitte an: mail@hlkroeber.de 